\title{
Article \\ A Novel Fault Feature Extraction Method for Bearing Rolling Elements Using Optimized Signal Processing Method
}

\author{
Weihan $\mathrm{Li}^{1}$, Yang $\mathrm{Li}^{2}$, Ling $\mathrm{Yu}^{3}$, Jian Ma ${ }^{4}$, Lei $\mathrm{Zhu}^{3}$, Lingfeng $\mathrm{Li}^{5}$, Huayue Chen ${ }^{6, *}$ and Wu Deng ${ }^{7, *}$ \\ 1 Engineering Training Center, Civil Aviation University of China, Tianjin 300300, China; whli@cauc.edu.cn \\ 2 Anhui CQC-CHEARI Technology Co., Ltd., Chuzhou 239000, China; liyang@cheari.com \\ 3 China Household Electric Appliance Research Institute, Beijing 100176, China; yul@cheari.com (L.Y.); \\ zhul@cheari.com (L.Z.) \\ 4 Chuzhou Technical Supervision and Testing Center, Chuzhou 239000, China; majian19909600139@163.com \\ 5 College of Marine Electrical Engineering, Dalian Maritime University, Dalian 116026, China; \\ lilingfeng1996@126.com \\ 6 School of Computer Science, China West Normal University, Nanchong 637002, China \\ 7 College of Electronic Information and Automation, Civil Aviation University of China, Tianjin 300300, China \\ * Correspondence: sunnyxiaoyue20@cwnu.edu.cn (H.C.); wdeng@cauc.edu.cn (W.D.)
}

Citation: Li, W.; Li, Y.; Yu, L.; Ma, J.; Zhu, L.; Li, L.; Chen, H.; Deng, W. A Novel Fault Feature Extraction Method for Bearing Rolling Elements Using Optimized Signal Processing Method. Appl. Sci. 2021, 11, 9095. https://doi.org/10.3390/app11199095

Academic Editor: Alberto Gatto

Received: 8 September 2021

Accepted: 27 September 2021

Published: 29 September 2021

Publisher's Note: MDPI stays neutral with regard to jurisdictional claims in published maps and institutional affiliations.

Copyright: (c) 2021 by the authors. Licensee MDPI, Basel, Switzerland. This article is an open access article distributed under the terms and conditions of the Creative Commons Attribution (CC BY) license (https:// creativecommons.org/licenses/by/ $4.0 /)$.

\begin{abstract}
A rolling element signal has a long transmission path in the acquisition process. The fault feature of the rolling element signal is more difficult to be extracted. Therefore, a novel weak fault feature extraction method using optimized variational mode decomposition with kurtosis mean (KMVMD) and maximum correlated kurtosis deconvolution based on power spectrum entropy and grid search (PGMCKD), namely KMVMD-PGMCKD, is proposed. In the proposed KMVMDPGMCKD method, a VMD with kurtosis mean (KMVMD) is proposed. Then an adaptive parameter selection method based on power spectrum entropy and grid search for MCKD, namely PGMCKD, is proposed to determine the deconvolution period $\mathrm{T}$ and filter order $\mathrm{L}$. The complementary advantages of the KMVMD and PGMCKD are integrated to construct a novel weak fault feature extraction model (KMVMD-PGMCKD). Finally, the power spectrum is employed to deal with the obtained signal by KMVMD-PGMCKD to effectively implement feature extraction. Bearing rolling element signals of Case Western Reserve University and actual rolling element data are selected to prove the validity of the KMVMD-PGMCKD. The experiment results show that the KMVMD-PGMCKD can effectively extract the fault features of bearing rolling elements and accurately diagnose weak faults under variable working conditions.
\end{abstract}

Keywords: rolling element; feature extraction; variational mode decomposition; maximum correlation kurtosis deconvolution; optimization method; kurtosis mean; variable conditions

\section{Introduction}

A rolling bearing is one of the core components of rotating machinery. Its normal operation is of great significance to the whole mechanical system [1-3]. Status monitoring and fault diagnosis have always been an important part of maintaining health [4-6]. The main fault types of rolling bearings include inner race faults, cage faults, rolling element faults, and outer race faults [7-11]. Because the rolling element is inside the rolling bearing, the transmission path of its fault signal is more complex, which makes the fault information of the rolling element fault signal is more difficult to be extracted [12-15]. Therefore, the research on the enhancement and the effective separation of fault features is the key step in fault diagnosis.

In the past few decades, many scholars have presented various signal processing methods to extract fault features from signals, such as wavelet transform (WT), empirical mode decomposition (EMD), local mean decomposition (LMD), and so on [16-24]. These methods are applied to better extract fault features from the signal, but they also have 
some shortcomings. WT needs to early select wavelet basis function before the signal is processed. The EMD and LMD have mode mixing. Although the ensemble EMD(EEMD) alleviates the mode mixing, the number of iterations will increase during signal processing, which results in an increase in the amount of calculation [25-31]. Therefore, Dragomiretskiy et al. [32] presented a VMD method in 2014. The central frequency and bandwidth of intrinsic mode functions (IMFs) are iteratively updated by solving variational problems to decompose signals into a series of IMFs. This method not only overcomes the mode mixing and end effect but also has suitable robustness. Therefore, the VMD has been extensively applied in fault diagnosis. Zhang et al. [33] used VMD to extract bearing fault features. However, the VMD requires to early set the parameter $\mathrm{K}$, and improper selection of $k$ will affect the decomposition effect [34]. Therefore, the proper selection of $\mathrm{K}$ is of great significance for the improvement of VMD. Zhu et al. [35] presented a dual-threshold method to obtain the number of decomposition modes and used this improved VMD to extract features of partial discharge signal. Pu et al. [36] presented a fault feature method based on VMD with a stationary wavelet. This method selects $\mathrm{K}$ by kurtosis criterion. However, the method using the kurtosis criterion to determine the decomposition mode number is not very accurate in practical application, and under strong noise interference, the VMD is not enough to extract weak fault features. McDonald et al. [37] presented MCKD in 2012. This method aims at maximizing the correlation kurtosis to highlight the periodic fault information submerged by noise in the signal. It has a suitable extraction effect on weak fault information. Tang et al. [38] used MCKD to extract the weak gear fault features. However, the deconvolution effect of MCKD is greatly affected by the values of $\mathrm{L}$ and $\mathrm{T}$. The adaptive selection of the two parameters will greatly improve the algorithm effect. Lv et al. [39] used the grid search to select two parameters adaptively, and Shannon entropy is used as the evaluation index. This improved MCKD method has a suitable result in processing fault signals of rolling bearings. In addition, the other some feature extraction methods and fault diagnosis methods are also proposed in recent years. Zhao et al. [40] presented a new fault feature extraction method based on integrating ensemble empirical mode decomposition, mode selection, and multi-scale fuzzy entropy to accurately diagnose faults. Deng et al. [41] presented a center frequency method of double thresholds to improve the variational mode decomposition (VMD) method for extracting the features of signals. Wang et al. [42] presented a multi-objective particle swarm optimization algorithm to optimize the parameters of VMD for the composite fault diagnosis of the gearbox. Shao et al. [43] presented a novel two-part approach: a stacked wavelet auto-encoder structure with a Morlet wavelet function for multisensory data fusion and a flexible weighted assignment of fusion strategies. At the same time, the performance of bearings can also be assessed with the use of measurements of such parameters as noise, vibrations, resistant torque [44]. In summary, the EMD, VMD, MCKD, and the other methods are proposed to extract fault features from signals to obtain better fault diagnosis results. However, These methods also exist some shortcomings, such as mode mixing, complex calculation, and so on.

In the strong noise environment, due to the long transfer path of the bearing rolling element signal, its features are weaker than those of other signals, which makes the fault features more difficult to be extracted. Therefore, the variational mode decomposition with kurtosis mean and maximum correlated kurtosis deconvolution based on power spectrum entropy and grid search are integrated to propose a novel weak fault feature extraction method, namely KMVMD-PGMCKD in this paper. In the proposed KMVMDPGMCKD method, the mode selection method based on kurtosis mean value is presented to tackle the mode selection problem of the VMD. Then, to solve the problem that the result processed by MCKD is affected by the $\mathrm{L}$ and $\mathrm{T}$, an adaptive parameter selection method using the power spectrum entropy and grid search is presented. Kurtosis mean is applied to determine the proper mode number $\mathrm{k}$ of the VMD, and then the VMD is employed to decompose the signal. Kurtosis is employed to select IMFs that contain more fault information and reconstruct the signal. The PGMCKD is employed to extract fault features 
of the reconstructed signals. The periodic shock components in IMFs will be enhanced while the noise will be suppressed. Bearing rolling element signal of Case Western Reserve University and actual rolling element data are selected to prove the validity of the proposed KMVMD-PGMCKD method.

\section{VMD with Kurtosis Mean (KMVMD)}

Dragomiretskiy introduced the algorithm called variational mode decomposition, which mainly uses signal processing tools of Hilbert transform, Wiener filtering, and frequency mixing. The signal is decomposed into different IMF by VMD [45]. The IMF is written as follows.

$$
u_{k}(t)=A_{k}(t) \cos \left(\phi_{k}(t)\right)
$$

The VMD requires to predefine the number of modes $k$. Improper $k$ will cause over decomposition or under decomposition. With the occurrence and development of bearing faults, the kurtosis value increases. The kurtosis value is higher, bearing fault is more serious. So, it is often applied to fix the number of mode $k$. After decomposition, the mode with the largest value is often selected. Its mathematical formula is shown as follows.

$$
k u r=\frac{E(x-\mu)^{4}}{\sigma^{4}}
$$

where $x$ indicates signal, $\mu$ indicates the mean value of $x, \sigma$ is the standard deviation of $x$.

However, it is not completely accurate to use the kurtosis criterion to fix the number of modes and choose the mode with the largest value for power spectrum analysis. This is because the fault frequency does not necessarily occur in the mode with the largest kurtosis under the strong noise interference but could occur in the mode with the other largest values.

Aiming at the shortage of kurtosis, VMD with kurtosis mean is proposed, which uses kurtosis mean to measure the mean value of fault information under different $k$. By calculating the kurtosis mean of the modes with larger kurtosis values at different $k$, the maximum kurtosis mean values at different $k$ are compared to obtain the number of modes. When the mode number is $k<5,\left[\frac{K}{2}\right]$ modes with larger values are chosen to calculate the kurtosis mean value. When the mode number $k \geq 5,3$ modes with larger values are chosen to calculate the kurtosis mean value. When $K$ is less than 5 , the problem of less mode number should be considered, so the number of selected IMF should be the number rounding half of $k$. This selection method can not only retain the fault information greatly under strong noise interference but also avoid the interference of abnormal modes on mode number selection. When $k \geq 5$, three modes are selected for analysis. Because for weak fault signals, the possibility of fault information appearing in IMF with the second or third larger value should be considered, but under normal conditions, fault information will not appear in modes with lower values. Therefore, the kurtosis mean value of the three larger kurtosis values of IMF can represent the proportion of fault information in the signal.

The expression of the kurtosis mean is shown in Equation (3).

$$
k_{\text {urmean }}=\frac{1}{N} \sum_{i=1}^{N} k u r_{i}
$$

where $N$ represents the number of IMFs.

The specific implementation steps of the KMVMD are given as follows.

Step 1: Determine the number of modes.

Step 2: Decompose fault signals using VMD in order to obtain IMFs in different $k$. $I_{k}=\left\{I M F_{i}\right\}\left(k=2, \ldots, k_{\text {max }}, i=1,2, \ldots, k\right)$;

Step 3: Calculate the kurtosis of each IMF, the set of kurtosis of IMF is obtained. $\operatorname{kur}_{k}=\left\{\operatorname{kur}\left(I M F_{i}\right)\right\}\left(k=2, \ldots, k_{\max }, i=1,2, \ldots, k\right)$; 
Step 4: In $k u r_{k}, j$ larger kurtosis is selected, so the set of larger kurtosis is obtained. $\operatorname{kur}_{k_{-} j}\left(k=2,3,4, j=1, \cdots,\left[\frac{k}{2}\right] ; k=5,6, \cdots, k_{\text {max }}, j=1,2,3\right)$. When $k<5, j=\left[\frac{k}{2}\right]$, When $k \geq 5, j=3$;

Step 5: Calculate the mean of larger kurtosis. kur_mean $k=\left\{\frac{1}{j_{\max }} \sum_{j=1}^{j_{\max }} k u r_{K_{-} j}\right\}$ $\left(k=2,3,4, j=1, \cdots,\left[\frac{k}{2}\right] ; k=5,6, \cdots, k_{\max }, j=1,2,3,\right)$;

Step 6: Finding the maximum value max_kurm meank in $\left\{k u r m \_m e a n_{k}\right\}$;

Step 7: The mode number corresponding to max_kur mean $K$ is the number of modes for VMD, and the modes with larger kurtosis values are chosen to obtain the reconstructed signal.

\section{MCMD with Power Spectral Entropy and Grid Search (PGMCKD)}

\section{1. $M C K D$}

$x(n)$ is periodic impulse signal, $h(n)$ is the transmission path response, and $e(n)$ is noise interference of the external environment. Then the measured signal $y(n)$ using sensor can be described as follow.

$$
y(n)=h(n) \cdot x(n)+e(n)
$$

In fact, the function of MCKD is to seek out an optimal FIR filter $f(n)$ to achieve the recovery of $x(n)$ by deconvolution; that is, the correlation kurtosis of the signal is maximized.

$$
x(n)=y(n) * f(n)
$$

The maximized correlation kurtosis value is given.

$$
C K_{M}(T)=\max _{f} \frac{\sum_{n=1}^{N}\left(\prod_{m=0}^{M} x_{n-m T}\right)^{2}}{\left(\sum_{n=1}^{N} x_{n}^{2}\right)^{M+1}}
$$

The Expression (4) is derivatized.

$$
\frac{d}{d f_{k}} C K_{M}(T)=0, k=1,2, \cdots, L
$$

Calculate the filter coefficients.

$$
f=\frac{\left\|x^{2}\right\|}{2\|B\|^{2}}\left(Y_{0} Y_{0}^{T}\right)^{-1} \sum_{m=0}^{M} Y_{m T} A_{m}
$$

where $T$ is the deconvolution period, $M$ represents the order shift. $f$ represents the finite impulse response filter vector, $L$ is the filter size. The coefficients in Expression (8) are obtained.

$$
\begin{gathered}
r=\left[\begin{array}{llllll}
0 & T & 2 T & \ldots & m T
\end{array}\right], A_{m}=\left[\begin{array}{c}
x_{1-m T}^{-1}\left(x_{1}^{2} x_{1-T}^{2} \ldots x_{1-m T}^{2}\right) \\
\vdots \\
x_{N-m T}^{-1}\left(x_{N}^{2} x_{N-T}^{2} \ldots x_{N-m T}^{2}\right)
\end{array}\right]_{N \times 1} B \\
=\left[\begin{array}{ccc}
x_{1} y_{1-T} & \cdots & x_{1-m T} \\
& \vdots & \\
x_{N} y_{N-T} & \cdots & x_{N-m T}
\end{array}\right]_{N \times 1}
\end{gathered}
$$

\subsection{Parameter Optimization of $M C K D$}

The $L$ and $T$ are two major parameters in MCKD, especially for $T$. The value of $T$ can determine the enhancement performance of MCKD. When MCKD is employed to enhance the signal, the optimal parameters for MCKD are determined according to the 
signal characteristics. The enhancement performance of the MCKD is decided by the value of parameters.

In theory, the value of $T$ can be determined.

$$
T=\frac{f_{s}}{f_{0}}
$$

where $f_{0}$ is fault frequency, $f_{s}$ is sampling frequency.

However, in practical application, the value of $\mathrm{T}$ is different from the theoretical value. Therefore, the value of $\mathrm{T}$ is optimized. Usually, the value range of $\mathrm{L}$ is from 100 to 500 . Due to the interaction between $\mathrm{L}$ and $\mathrm{T}$, it is of major significance to study the adaptive selection of $\mathrm{L}$ and $\mathrm{T}$ for improving the enhancement performance of MCKD.

If the sampling frequency $y(n)$ is $f_{s}$. The sampling points are $N . Y\left(\omega_{i}\right)$ is obtained by Fourier transform. $\omega_{i}=\frac{2 \pi f_{s}}{N} i(i=1,2, \cdots, N / 2)$

The formula of power spectral density of signal is as follows.

$$
p\left(\omega_{i}\right)=\frac{\left|Y\left(\omega_{i}\right)\right|^{2}}{N}
$$

$p\left(\omega_{i}\right)$ was normalized to $p_{i}$.

$$
p_{i}=\frac{p\left(\omega_{i}\right)}{\sum_{i} p\left(\omega_{i}\right)}
$$

Power spectral entropy can effectively reflect the distribution characteristics of signal energy, so it is applied to optimize parameters. The expression of power spectrum entropy is described as follows.

$$
H=-\sum_{i} p_{i} * \log p_{i}
$$

The grid search method is a search algorithm to specify parameter values. The possible values of various parameters are arranged and combined. The possible combination results are shown to produce a "grid". It exists a low precision requirement, and the step size can improve the calculation speed. The search range of $L$ is $[a, b]$, step is $n_{1}$. The search range of $T$ is $[c, d]$, step is $n_{2}$. The possible values of $L$ and $T$ are arranged, and the generated grid is considered as $(L, T) \in[a, b] \times[c, d]$.

The optimization evaluation of parameters using power spectral entropy is described as follows.

$$
\min _{(L, T) \in[a, b] \times[c, d]}-\sum_{i} p_{i}(L, T) * \log _{2}\left(p_{i}(L, T)\right)
$$

where $i=1,2, \ldots, n$.

\section{A Fault Feature Extraction Method}

Because it is difficult to extract fault features of rolling elements under complex working conditions and strong interference, a new feature extraction method of rolling element fault based on KMVMD and PGMCKD, namely KMVMD-PGMCKD, is proposed here. Firstly, the KMVMD is employed to decompose the signal into IMFs and reconstruct the signal. Then, based on the parameter adaptive selection method of power spectrum entropy, the $L$ and $T$ of MCKD are selected adaptively, and the fault features of the reconstructed signal are effectively enhanced by PGMCKD. Finally, the power spectrum is selected to analyze the deconvoluted signal to diagnose faults.

The realized steps of the KMVMD-PGMCKD are described as follows.

Step 1: Determine the maximum number of modes and decompose the signal.

Step 2: Based on the maximum kurtosis mean value, the mode number is determined, and IMFs with larger kurtosis values are selected for reconstructing the signal.

Step 3: Set the values of $L$ and $T$ in MCKD. The interference of rotating frequency is considered to determine $T$. Calculate the theoretical value of the search range, and obtain the search range. 
Step 4: Grid search is employed to determine two parameters in MCKD, and the power spectral entropy evaluation criterion is employed to obtain ideal values of parameters.

Step 5: Hilbert transform is employed to transform the enhanced signal. The power spectrum is applied to analyze the enhanced signal to identify fault feature frequency.

\section{The Experiment and Analysis}

To prove the effectiveness of KMVMD-PGMCKD, bearing rolling element fault signals from Case Western Reserve University and bearing rolling element fault signals from QPZZ-П platform are selected in this paper.

\subsection{Fault Data from CWRU}

The experiment is to analyze the bearing rolling element fault signal of Case Western Reserve University [45]. The bearing is SKF6205, the pitch diameter is $39.04 \mathrm{~mm}$, there are nine rolling elements with a diameter of $7.94 \mathrm{~mm}$, the speed is $1797 \mathrm{r} / \mathrm{min}$, and the load is $0 \mathrm{HP}$, the sampling frequency is $12,000 \mathrm{~Hz}$. The signal length is 4096 . By theoretical calculation, the fault frequency $f_{0}$ of bearing rolling element is $141.1 \mathrm{~Hz}$, and the rotating frequency $f_{r}$ is $29.2 \mathrm{~Hz}$.

The time waveform and power spectrum are shown in Figure 1.

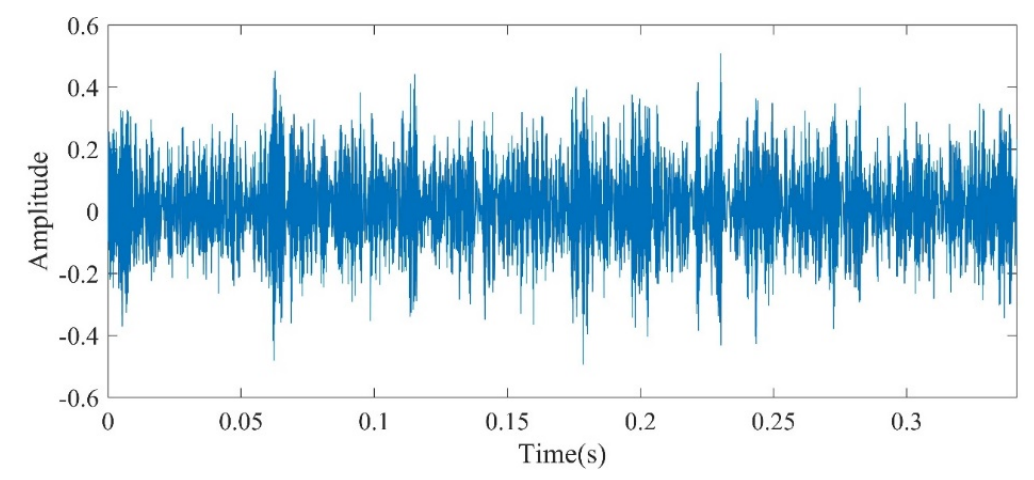

(a) Time waveform

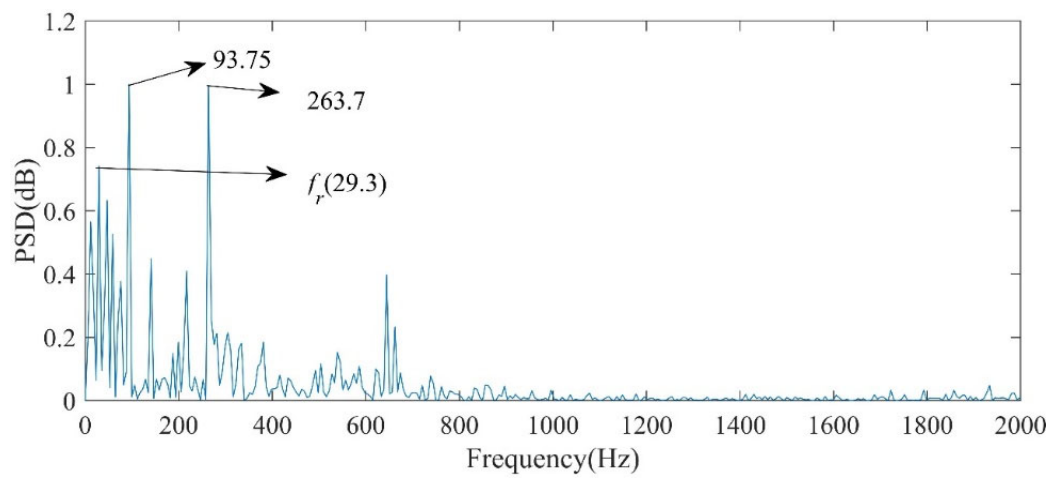

(b) Power spectrum

Figure 1. The time waveform and power spectrum of rolling element signal.

It cannot be found any useful information for the rolling element from Figure 1. To extract fault features from fault signal, the KMVMD-PGMCKD is employed to process the rolling element fault signal.

\subsubsection{Experimental Analysis}

Firstly, KMVMD is employed to select the mode number of VMD. The fault signals are decomposed in different scales, and the kurtosis and the kurtosis mean values are calculated, which are shown in Table 1. 
Table 1. Kurtosis and kurtosis mean values of each IMFs with different $k$.

\begin{tabular}{cccccccccc}
\hline & IMF1 & IMF2 & IMF3 & IMF4 & IMF5 & IMF6 & IMF7 & $\begin{array}{c}\text { Kurtosis } \\
\text { Mean }\end{array}$ \\
\hline 2 & 3.0346 & $\mathbf{3 . 0 4 0 6}$ & & & & & & 3.0406 \\
3 & 3.0427 & $\mathbf{3 . 1 2 7 9}$ & 3.0361 & & & & & 3.0853 \\
4 & 2.5209 & 2.118 & $\mathbf{3 . 1 2 0 5}$ & $\mathbf{3 . 0 3 6 5}$ & & & & 3.0785 \\
5 & 2.519 & 2.1155 & $\mathbf{3 . 0 9 7 4}$ & $\mathbf{3 . 4 1 1 7}$ & $\mathbf{2 . 7 4 9 9}$ & & & 3.0863 \\
6 & 2.3611 & 2.9584 & $\mathbf{3 . 1 0 0 4}$ & $\mathbf{3 . 4 0 0 3}$ & 2.7476 & $\mathbf{3 . 1 5 1 6}$ & & $\mathbf{3 . 2 1 7 4}$ \\
7 & 2.3452 & 3.0009 & 1.9672 & $\mathbf{3 . 0 9 0 1}$ & $\mathbf{3 . 4 0 8 3}$ & 2.7464 & $\mathbf{3 . 1 0 5 5}$ & 3.2013 \\
\hline
\end{tabular}

The bold values in Table 1 are the kurtosis values used to compute the mean value. According to the data, the IMF with a larger kurtosis value is selected for signal reconstruction when $k=6$, which are IMF3, IMF4, and IMF6. The power spectrum of the reconstructed signal is given in Figure 2.

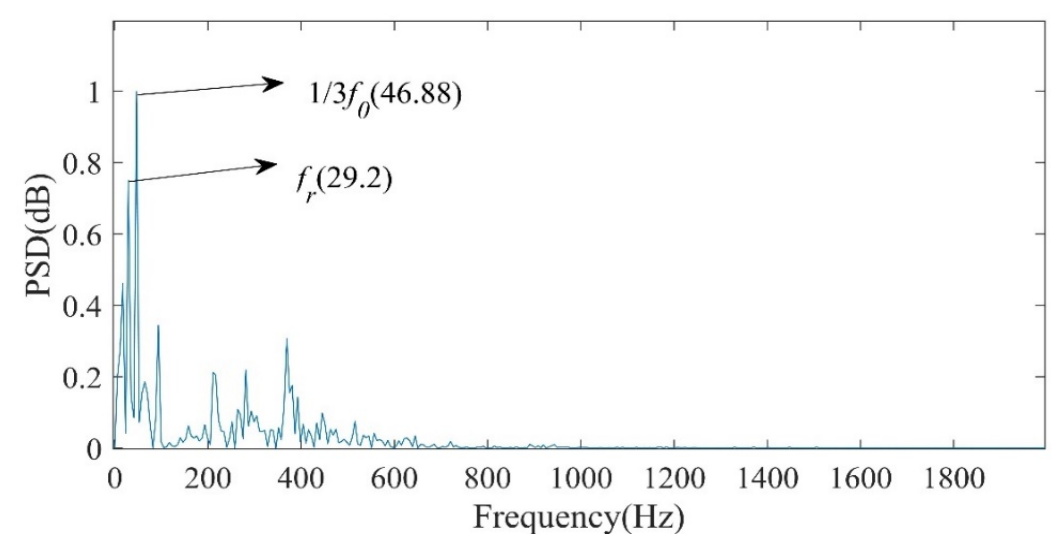

Figure 2. Power spectrum of reconstructed signal.

It can be clearly found that $1 / 3$ harmonic of fault frequency $(46.88 \mathrm{~Hz})$ and rotating frequency $(29.2 \mathrm{~Hz})$ are obvious in Figure 2, which are better than the original signal without processing. However, since the fault frequency is not found directly in Figure 2, in order to obtain a better result, the reconstructed signal will be further processed by PGMCKD.

Firstly, the calculated theoretical value of $T$ is 85 according to Equation (10), so the search range of $T$ is set $[83,87]$, the step size is 1 , the search range of $L$ is set $[100,500]$, its step size is 5 . Based on the evaluation criterion of power spectrum entropy, the optimized parameter by the grid search method is $[T, L]=[87,460]$. The power spectrum of the deconvoluted signal by PGMCKD is given in Figure 3.

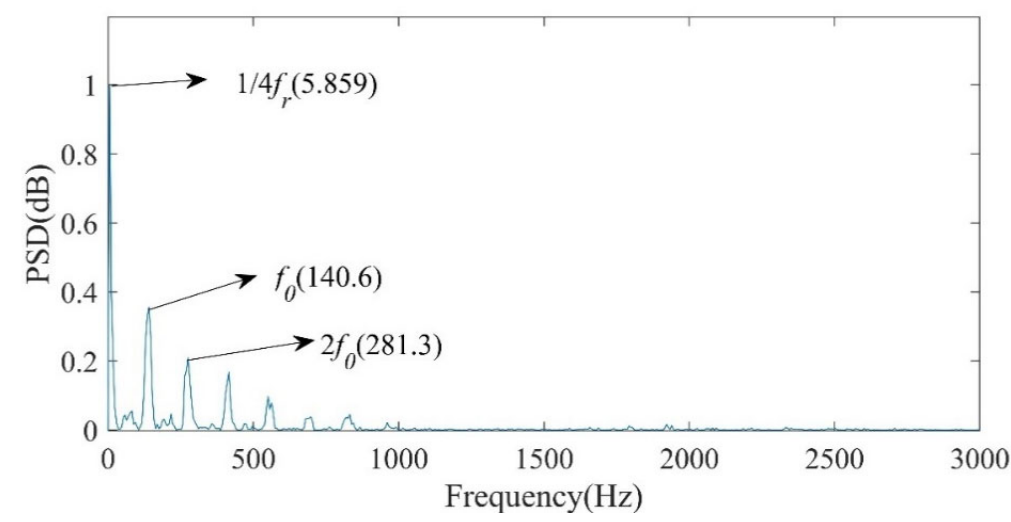

Figure 3. Power spectrum of element fault signal by KMVMD-PGMCKD. 
From Figure 3, it can be clearly found that $1 / 5$ harmonic of the rotating frequency $(5.859 \mathrm{~Hz})$, the fault frequency $f_{0}(140.6 \mathrm{~Hz})$, and its second harmonic $(281.3 \mathrm{~Hz})$ are obvious. It can be seen that the KMVMD-PGMCKD can accurately extract fault features of the bearing rolling element signal.

\subsubsection{Comparison Analysis}

To prove the effectiveness of PGMCKD, the MCKD and MCKD based on Shannon entropy are selected to compare with PGMCKD. For MCKD, the value of $T$ is 85 , and $L$ can be any value from 100 to 500 . In this experiment, the value of $L$ is 460 , which is the same as PGMCKD. The Shannon entropy optimization parameter method sets the value range of $T$ as $[83,87]$, the step size as 1 , the search range of $L$ as $[100,500]$, the step size is 5 , and the final result of $[L, T]$ is $[87,325]$. The power spectrum by different methods is given in Figures 4 and 5.

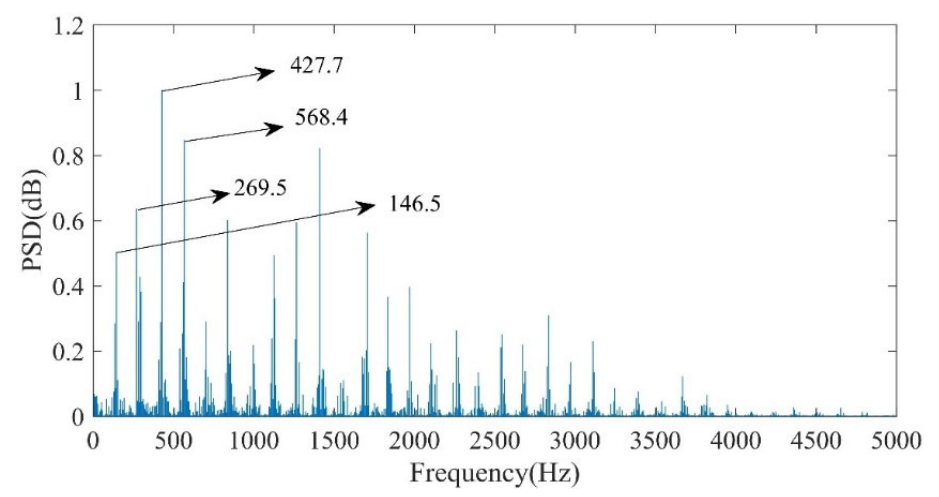

Figure 4. Power spectrum of the reconstructed signal by MCKD.

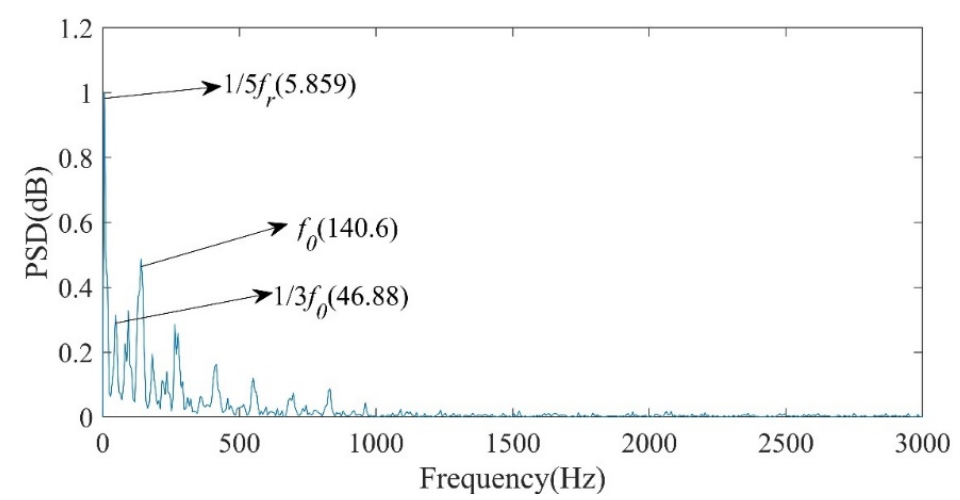

Figure 5. Power spectrum of the reconstructed signal by MCKD based on Shannon entropy.

From Figure 4, after the reconstructed signal is processed by MCKD, the extracted fault features are still weak, so they cannot meet the requirements of fault diagnosis. In Figure 6 , it can be seen $1 / 5$ harmonic of the rotating frequency $(5.859 \mathrm{~Hz})$, and the fault frequency $f_{0}(140.6 \mathrm{~Hz})$ and its $1 / 3$ harmonic $(46.88 \mathrm{~Hz})$. However, compared with Figure 4, the power spectrum of the signal processed by PGMCKD has less frequency component of noise, and the effect is more obvious. Therefore, the experimental comparison results can prove the effectiveness of PGMCKD for fault diagnosis.

\subsubsection{Experimental Results under Variable Condition}

To prove the effectiveness of KMVMD-PGMCKD under variable working conditions, the experiments are carried out under the conditions of $2 \mathrm{HP}$ motor load and $1748 \mathrm{r} / \mathrm{min}$ rotating speed. According to the changed conditions, the fault frequency is about $137.4 \mathrm{~Hz}$, and the rotating frequency is about $29.1 \mathrm{~Hz}$. The experimental results are shown in Figure 6. 


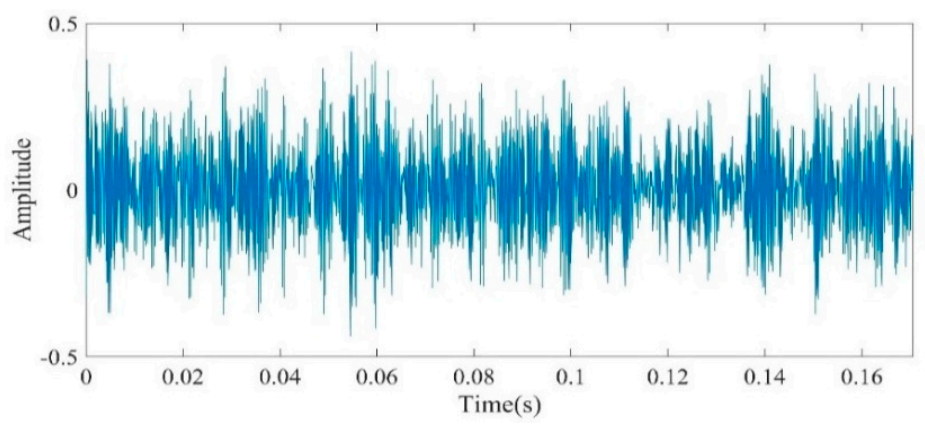

(a) Time waveform

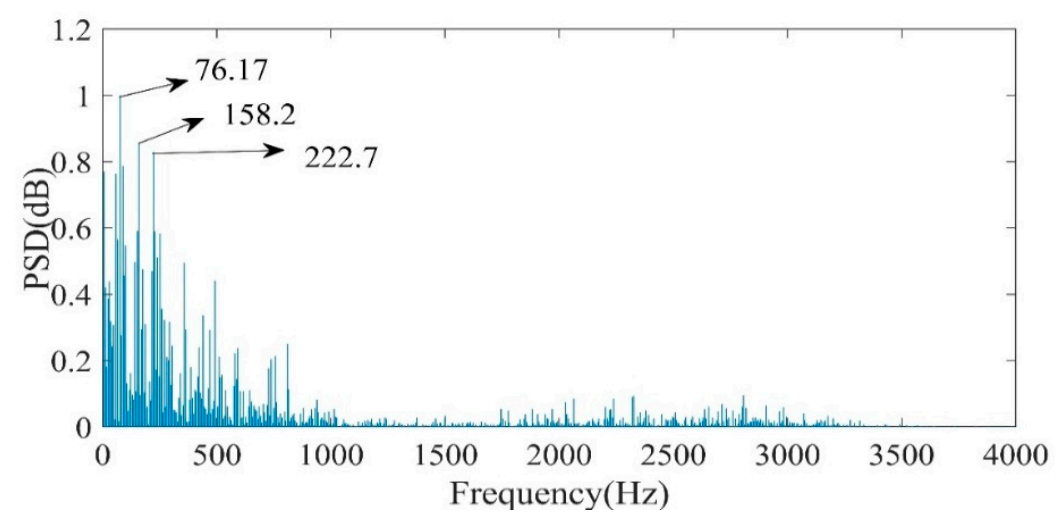

(b) Power spectrum of fault signal

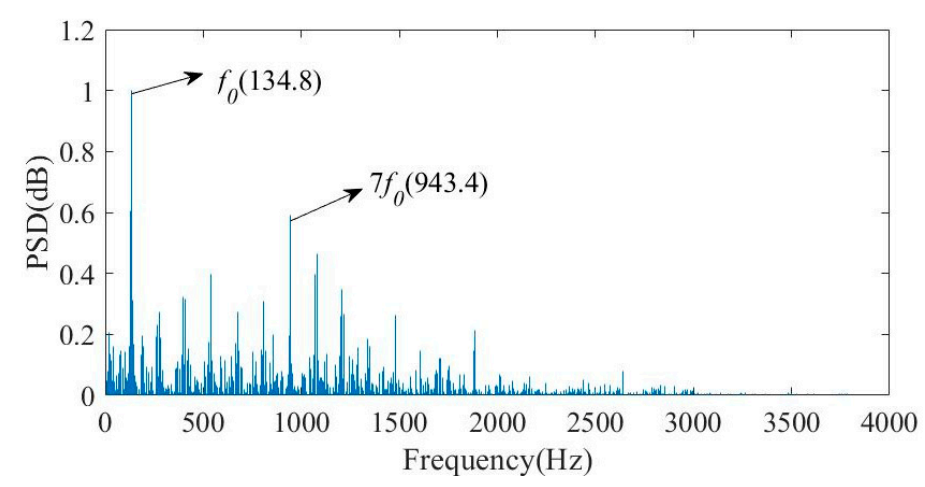

(c) Power spectrum of processed signal

Figure 6. The obtained results of rolling element fault signal under variable working conditions.

From Figure 6, the fault frequency $f_{0}(134.8 \mathrm{~Hz})$ and $7 f_{0}(943.4 \mathrm{~Hz})$ are obvious. Therefore, the experiment results proved that the KMVMD-PGMCKD still can extract the fault features under variable working conditions.

\subsection{Fault Data from QPZZ-П}

To further prove the effectiveness of the KMVMD-PGMCKD, the fault signal from QPZZ-II rolling bearing is selected here. The bearing is N205, the pitch diameter is $39 \mathrm{~mm}$, rolling elements with a diameter of $7.5 \mathrm{~mm}$ are 13 , the speed is $1500 \mathrm{r} / \mathrm{min}$, and the sampling frequency is $12 \mathrm{kHz}$. The signal length is 2048. Therefore, the fault characteristic frequency is $125.2 \mathrm{~Hz}$, the inner race fault characteristic frequency is $194.2 \mathrm{~Hz}$ and the rotating frequency $f_{r}$ is $25 \mathrm{~Hz}$.

The time waveform and power spectrum are given in Figure 7. 


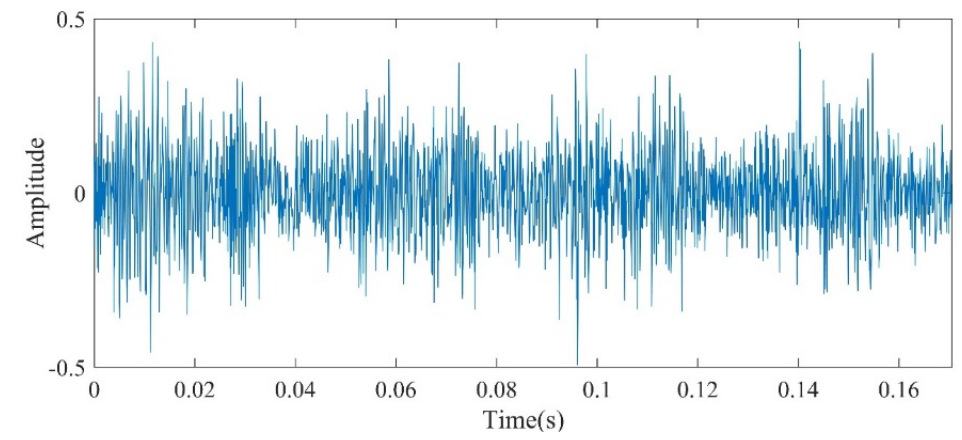

(a) Time waveform

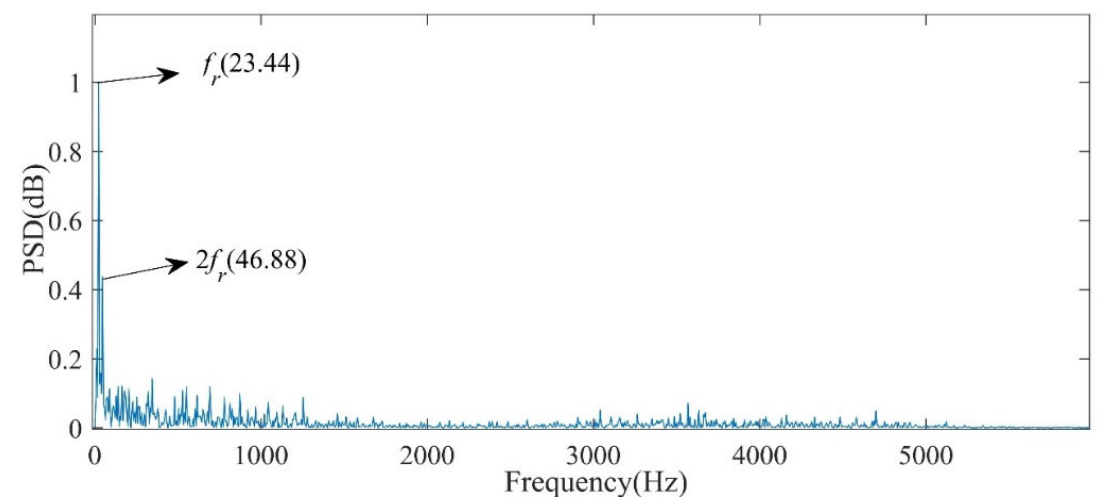

(b) Power spectrum

Figure 7. The obtained experiment results.

From Figure $7 \mathrm{a}$, the signal contains strong noise interference, and faults are not obvious. In Figure $7 \mathrm{~b}$, the rotating frequency and its second harmonic are only obviously obtained, and the amplitude of other components is relatively weak, so it is difficult to find any useful features for fault diagnosis. The KMVMD-PGMCKD is used to further process in order to extract fault features.

\subsubsection{Experimental Analysis}

Firstly, the KMVMD is employed to select the mode number. The fault signal is decomposed in different scales, and the kurtosis value and the kurtosis mean value are calculated, which are given in Table 2.

Table 2. Kurtosis and kurtosis mean value of each IMF with different $k$.

\begin{tabular}{|c|c|c|c|c|c|c|c|c|c|}
\hline $\mathbf{k}$ & Kurtosis & IMF1 & IMF2 & IMF3 & IMF4 & IMF5 & IMF6 & IMF7 & $\begin{array}{c}\text { Kurtosis } \\
\text { Mean }\end{array}$ \\
\hline & 2 & 4.3737 & 4.2419 & & & & & & 4.3737 \\
\hline & 3 & 4.3696 & 4.2406 & 4.8543 & & & & & 4.6119 \\
\hline & 4 & 4.2420 & 4.3257 & 5.7181 & 4.8536 & & & & 5.2850 \\
\hline & 5 & 3.6537 & 3.5656 & 4.4510 & 5.4256 & 4.8390 & & & 4.9052 \\
\hline & 6 & 3.3937 & 3.5400 & 4.4029 & 5.7447 & 5.3062 & 4.8391 & & 5.2960 \\
\hline & 7 & 3.3374 & 4.3578 & 4.3320 & 5.7153 & 5.6530 & 5.2321 & 3.4010 & 5.5334 \\
\hline
\end{tabular}

According to the data in the table, IMF with a larger kurtosis value is chosen to reconstruct the signal under $k=7$, which are IMF4, IMF5, and IMF6. The power spectrum of the reconstructed signal is given in Figure 8.

It can be clearly found that $1 / 3$ fault frequency harmonic $(41.02 \mathrm{~Hz})$ and rotating frequency $(23.44 \mathrm{~Hz})$ are obvious in Figure 8. Then the PGMCKD is applied to further deal with the reconstructed signal. The calculated theoretical value of $T$ is 96 according 
to Equation (10), the search range of $T$ is set [94, 98], the step size is 1 , the search range of $L$ is set $[100,500]$, its step size is 5 . Based on the evaluation criterion of power spectrum entropy, the grid search is employed to obtain optimal parameter $[T, L]=[97,175]$. The power spectrum of the deconvoluted signal by PGMCKD is given in Figure 9.

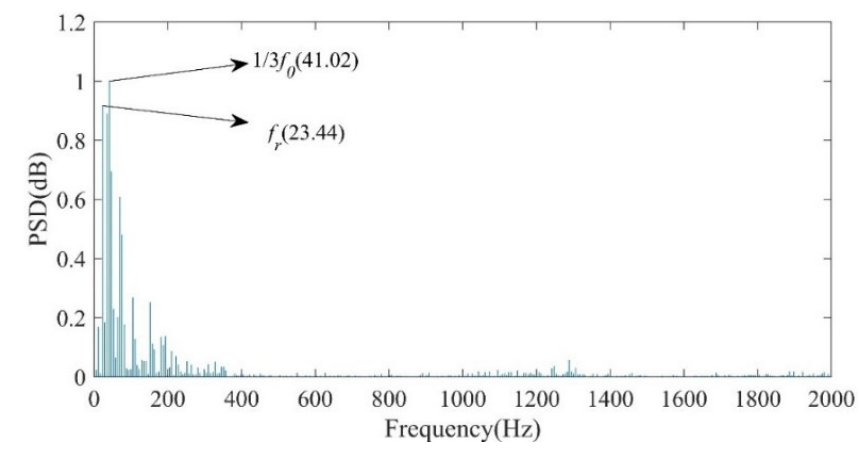

Figure 8. Power spectrum of reconstructed signal.

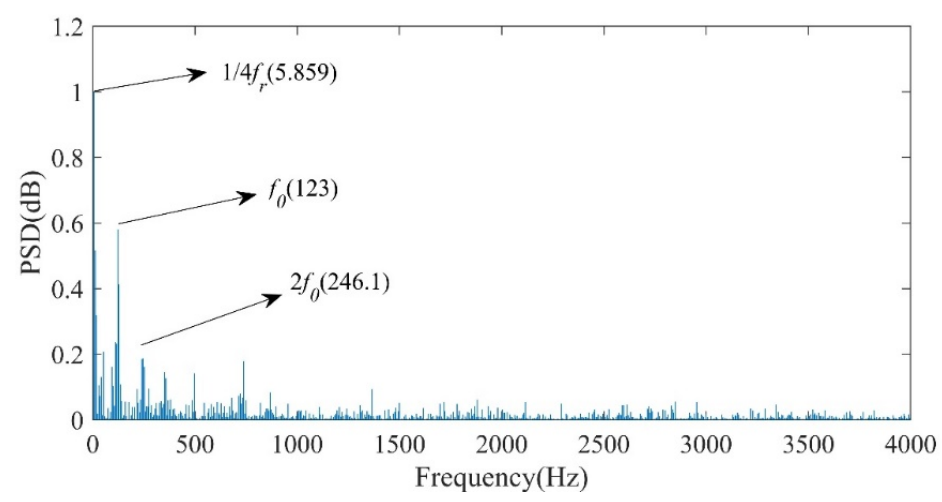

Figure 9. Power spectrum by KMVMD-PGMCKD.

From Figure 10, it can be clearly found that $1 / 4$ harmonic of the rotating frequency $(5.859 \mathrm{~Hz})$, the fault frequency $f_{0}(140.6 \mathrm{~Hz})$, and its second harmonic $(281.3 \mathrm{~Hz})$. It further proved that KMVMD-PGMCKD can accurately extract fault features of the rolling element signal.

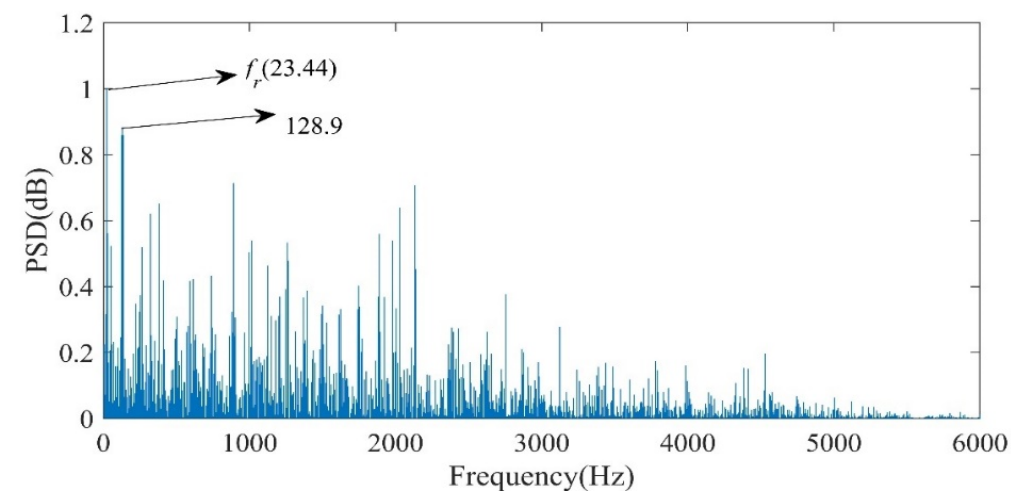

Figure 10. Power spectrum of reconstructed signal by MCKD.

\subsubsection{Comparison Analysis}

To verify the effectiveness of PGMCKD, the MCKD and MCKD based on Shannon entropy are selected to compare with PGMCKD. For MCKD, the theoretical value of $T$ is 96, the value of $L$ is 175 . Shannon entropy is used to set the value range of $T$ as [94, 98], 
the step size is 1 , the search range of $L$ is $[100,500]$, the step size is 5 , and the final result of $[L, T]$ is $[98,395]$. The processed signal power spectrum is given in Figures 10 and 11.

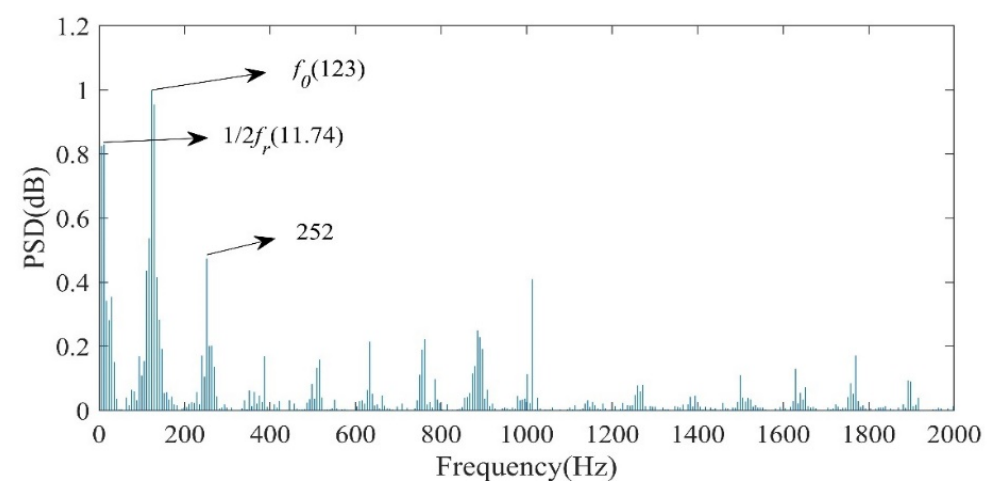

Figure 11. Power spectrum of the reconstructed signal by MCKD based on Shannon entropy.

In Figure 11, after the MCKD is employed to deal with the reconstructed signal, the fault features still cannot be extracted. In Figure 5, fault frequency $f_{0}(123 \mathrm{~Hz})$ has the largest amplitude, and $1 / 2$ harmonic of the rotating frequency $(11.74 \mathrm{~Hz})$ can also be found. However, compared with Figure 9, harmonic of the fault frequency occurs. Therefore, the experimental comparison results prove the effectiveness of PGMCKD.

\subsubsection{Experimental Results under Variable Working Condition}

In order to verify the effectiveness of KMVMD-PGMCKD under variable working conditions, the experiment is carried out again after the rotating speed is changed to $1000 \mathrm{r} / \mathrm{min}$. According to the changed conditions, the fault frequency is about $83.5 \mathrm{~Hz}$, and the rotating frequency is about $16.7 \mathrm{~Hz}$. The experimental results are given in Figure 12.

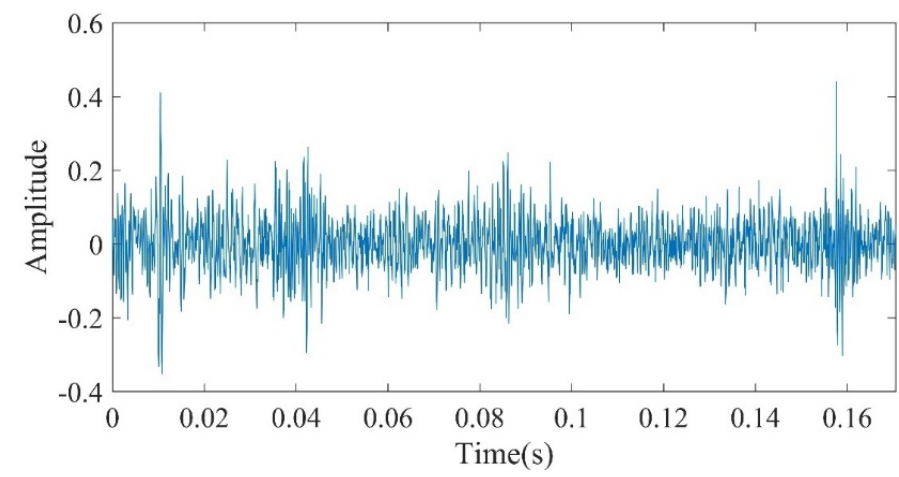

(a) Time waveform of fault signal

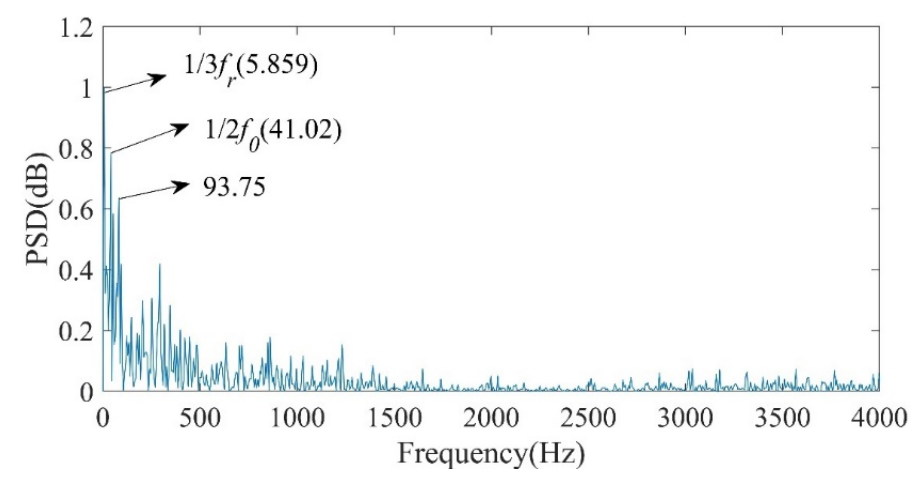

(b) Power spectrum of fault signal

Figure 12. Cont. 


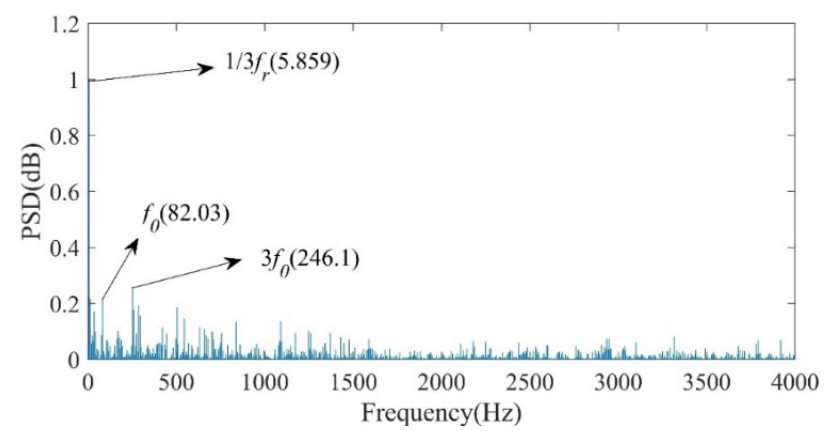

(c) Power spectrum of processed signal

Figure 12. The obtained results under variable working conditions.

From Figure 12, fault frequency $f_{0}(82.03 \mathrm{~Hz})$ and its third harmonic $(246.1 \mathrm{~Hz})$ under the condition of variable working conditions can still be extracted from the fault signal of the rolling element by KMVMD-PGMCKD, which further proves that this method has strong generalization ability under the variable working condition.

\section{Conclusions}

Because it is difficult to extract fault features from rolling element signals, a novel feature extraction method called KMVMD-PGMCKD is proposed for bearing rolling elements. The KMVMD provides a new mode number selection method to improve the signal processing effect of VMD. The PGMCKD provides a new parameter optimization method to improve the signal processing effect of MCKD. Through the experimental analysis under different working conditions, the following conclusions are obtained:

(1). The kurtosis mean has a suitable effect on the selection of mode number, which avoids the phenomenon of over decomposition or under decomposition and provides a suitable foundation for further processing;

(2). The power spectrum entropy is used as the evaluation criterion for the selection of parameters in MCKD. The difference between the actual and theoretical conditions is considered. The $\mathrm{L}$ and the $\mathrm{T}$ are adaptively selected, which improves the effect of MCKD on the enhancement of weak fault features;

(3). The combination of improved VMD and MCKD fully suppresses the noise interference on the fault features, highlights the periodic impact component, and solves difficult fault feature problems for bearing rolling elements. The effectiveness of the KMVMDPGMCKD is also verified by experiments;

(4). The signal processing effect of KMVMD-PGMCKD is not affected by the variable working condition. The experiment shows that KMVMD-PGMCKD has a strong generalization ability under the variable working condition.

Author Contributions: Methodology, W.L. and H.C.; validation, Y.L. and L.Z.; resources, L.Y.; data curation, J.M.; writing—original draft preparation, W.L., Y.L. and L.L; writing—review and editing, W.D.; project administration, L.L.; funding acquisition, W.D. and Y.L. All authors have read and agreed to the published version of the manuscript.

Funding: This research was funded by the Research Foundation for Civil Aviation University of China (2020KYQD123).

Institutional Review Board Statement: Not applicable.

Informed Consent Statement: Not applicable.

Data Availability Statement: https:/ / csegroups.case.edu/bearingdatacenter/pages/welcome-casewestern-reserve-university-bearing-data-center-website (accessed on 10 May 2020).

Conflicts of Interest: The authors declare no conflict of interest. 


\section{References}

1. Liu, C.L.; Wu, Y.J.; Zhen, C.G. Rolling bearing fault diagnosis based on variational mode decomposition and fuzzy c-means clustering. Proc. Chin. Soc. Electr. Eng. 2015, 35, 3358-3365.

2. Jin, T.; Xia, H.; Chen, H. Optimal control problem of the uncertain second-order circuit based on first hitting criteria. Math. Methods Appl. Sci. 2021, 44, 882-900. [CrossRef]

3. Li, B.; Chow, M.Y.; Tipsuwan, Y.; Hung, J.C. Neural-network-based motor rolling bearing fault diagnosis. IEEE Trans. Ind. Electron. 2000, 47, 1060-1069. [CrossRef]

4. Zheng, J.; Yuan, Y.; Zou, L.; Deng, W.; Guo, C.; Zhao, H.M. Study on a novel fault diagnosis method based on VMD and BLM. Symmetry 2019, 11, 747. [CrossRef]

5. Guo, S.; Zhang, X.; Du, Y.; Zheng, Y.; Cao, Z. Path planning of coastal ships based on optimized DQN reward function. J. Mar. Sci. Eng. 2021, 9, 210. [CrossRef]

6. Xu, Y.; Chen, H.; Luo, J.; Zhang, Q.; Jiao, S.; Zhang, X. Enhanced Moth-flame optimizer with mutation strategy for global optimization. Inf. Sci. 2019, 492, 181-203. [CrossRef]

7. Huang, N.E.; Shen, Z.; Long, S.R.; Wu, M.C.; Shih, H.H.; Zheng, Q.; Yen, N.C.; Tung, C.C.; Liu, H.H. The empirical mode decomposition and the Hilbert spectrum for nonlinear and non-stationary time series analysis. Proc. R. Soc. Lond. 1998, 454, 903-995. [CrossRef]

8. Tse, P.W.; Peng, Y.H.; Yam, R. Wavelet analysis and envelope detection for rolling element bearing fault diagnosis-Their effectiveness and flexibilities. J. Vib. Acoust. 2001, 123, 303-310. [CrossRef]

9. Jin, T.; Xia, H.; Deng, W.; Li, Y.; Chen, H. Uncertain fractional-order multi-objective optimization based on reliability analysis and application to fractional-order circuit with Caputo type. Circuits Syst. Signal Process. 2021, 1-28. [CrossRef]

10. Li, T.Y.; Shi, J.Y.; Zhang, D.H. Color image encryption based on joint permutation and diffusion. J. Electron. Imaging 2021, 30, 013008. [CrossRef]

11. Rasolofondraibe, L.; Pottier, B.; Marconnet, P.; Perrin, E. Numerical model of the capacitive probe's capacitance for measuring the external loads transmitted by the bearing's rolling elements of rotating machines. IEEE Sens. J. 2013, 13, 3067-3072. [CrossRef]

12. Nikolaou, N.G.; Antoniadis, I.A. Rolling element bearing fault diagnosis using wavelet packets. NDTEE Int. 2002, 35, 197-205. [CrossRef]

13. Yang, Y.; Chen, J.S. A roller bearing fault diagnosis method based on EMD energy entropy and ANN. J. Sound Vib. 2006 294, 269-277.

14. Eren, L.; Devaney, M.J. Bearing damage detection via wavelet packet decomposition of the stator current. IEEE Trans. Instrum. Meas. 2004, 53, 431-436. [CrossRef]

15. Jin, T.; Ding, H.; Li, B.; Xia, H.; Xue, C. Valuation of interest rate ceiling and floor based on the uncertain fractional differential equation in Caputo sense. J. Intell. Fuzzy Syst. 2021, 40, 5197-5206. [CrossRef]

16. Zhao, H.M.; Liu, H.D.; Jin, Y.; Xue, C.X. Feature extraction for data-driven remaining useful life prediction of rolling bearings. IEEE Trans. Instrum. Meas. 2021, 70, 1-10. [CrossRef]

17. Li, X.; Yang, Y.; Shao, H.D.; Zhong, X.; Cheng, J. Symplectic weighted sparse support matrix machine for gear fault diagnosis. Measurement 2021, 168, 108392. [CrossRef]

18. Li, T.Y.; Zhou, M. ECG classification using wavelet packet entropy and random forests. Entropy 2016, 18, 285. [CrossRef]

19. Deng, W.; Shang, S.F.; Cai, X.; Zhao, H.M.; Xu, J.J. An improved differential evolution algorithm and its application in optimization problem. Soft Comput. 2021, 7, 5277-5298. [CrossRef]

20. Rai, V.K.; Mohanty, A.R. Bearing fault diagnosis using FFT of intrinsic mode functions in Hilbert-Huang transform. Mech. Syst. Signal Process. 2007, 21, 2607-2615. [CrossRef]

21. Mandic, D.P.; Rehman, N.U.; Wu, Z.; Huang, N.E. Empirical mode decomposition-based time-frequency analysis of multivariate signals: The power of adaptive data analysis. IEEE Signal Process. Mag. 2013, 30, 74-86. [CrossRef]

22. Cheng, J.; Yang, Y.; Yang, Y. A rotating machinery fault diagnosis method based on local mean decomposition. Digit. Signal Process. 2012, 22, 356-366. [CrossRef]

23. Guo, W.; Peter, W.T. A novel signal compression method based on optimal ensemble empirical mode decomposition for bearing vibration signals. J. Sound Vib. 2013, 332, 423-441. [CrossRef]

24. Wu, Y.J.; Zhen, C.G.; Liu, C.L. Application of variational mode decomposition in wind power fault diagnosis. Mech. Transm 2015, 129-132.

25. Deng, W.; Shang, S.; Cai, X.; Zhao, H.M.; Zhou, Y.Q.; Chen, H.Y.; Deng, W.Q. Quantum differential evolution with cooperative coevolution framework and hybrid mutation strategy for large scale optimization. Knowl.-Based Syst. 2021, 224, 107080. [CrossRef]

26. Zhao, H.; Li, D.; Deng, W.; Yang, X. Research on vibration suppression method of alternating current motor based on fractional order control strategy. Proc. Inst. Mech. Eng. Part E J. Process. 2017, 231, 786-799. [CrossRef]

27. Jin, T.; Ding, H.; Xia, H.; Bao, J. Reliability index and Asian barrier option pricing formulas of the uncertain fractional first-hitting time model with Caputo type. Chaos Solitons Fractals 2021, 142, 110409. [CrossRef]

28. Wu, H.; Liang, S.; Zou, L. Method of evaluating aerodynamic damping of tall buildings based on wavelet analysis. J. Vib. Shock 2008, 4, 93-96.

29. Jin, T.; Yang, X.; Xia, H.; Ding, H. Reliability index and option pricing formulas of the first hitting time model based on the uncertain fractional-order differential equation with Caputo type. Fractals 2021, 29, 2150012. [CrossRef] 
30. Chen, H.; Zhang, Q.; Luo, J.; Xu, Y.; Zhang, X. An enhanced Bacterial Foraging Optimization and its application for training kernel extreme learning machine. Appl. Soft Comput. 2020, 86, 105884. [CrossRef]

31. Dragomiretskiy, K.; Zosso, D. Variational mode decomposition. IEEE Tran Signal Process. 2014, 62, 531-544. [CrossRef]

32. Zhang, M.; Jiang, Z.; Feng, K. Research on variational mode decomposition in rolling bearings fault diagnosis of the multistage centrifugal pump. Mech. Syst. Signal Process. 2017, 93, 460-493. [CrossRef]

33. Miao, Y.; Zhao, M.; Lin, J. Identification of mechanical compound-fault based on the improved parameter-adaptive variational mode decomposition. ISA Trans. 2019, 84, 82-95. [CrossRef] [PubMed]

34. Zhu, Y.L.; Jia, Y.F.; Wang, L.W.; Li, L.; Zheng, Y. Feature extraction and classification on partial discharge signals of power transformers based on improved variational mode decomposition and Hilbert transform. Trans. China Electrotech. Soc. 2017, 32, 221-235.

35. Pu, Z.X.; Yin, H.; Zhang, N.; Yan, W.; Peng, Z. Bearing fault diagnosis using vmd and stationary wavelet method based on kurtosis criterion. Mach. Des. Res. 2017, 33, 67-71.

36. Mcdonald, G.L.; Zhao, Q.; Zuo, M.J. Maximum correlated kurtosis deconvolution and application on gear tooth chip fault detection. Mech. Syst. Signal Process. 2012, 33, 237-255. [CrossRef]

37. Tang, G.J.; Wang, X.L. Weak feature extraction of gear fault based on maximum correlated kurtosis deconvolution and sparse code shrinkage. J. Vib. Eng. 2015, 28, 478-486.

38. Zhongliang, L.; Tang, B.; Zhou, Y.; Meng, J. Rolling bearing early fault diagnosis based on maximum correlated kurtosis deconvolution optimized with grid search algorithm. J. Vib. Shock 2016, 35, 29-34.

39. Zhao, H.M.; Sun, M.; Deng, W.; Yang, X. A new feature extraction method based on EEMD and multi-scale fuzzy entropy for motor bearing. Entropy 2016, 19, 14. [CrossRef]

40. Deng, W.; Liu, H.; Zhang, S.; Liu, H.; Zhao, H.; Wu, J. Research on an adaptive variational mode decomposition with double thresholds for feature extraction. Symmetry 2018, 10, 684. [CrossRef]

41. Wang, Z.; He, G.; Du, W.; Zhou, J.; Kou, Y. Application of parameter optimized variational mode decomposition method in fault diagnosis of gearbox. IEEE Access 2019, 7, 44871-44882. [CrossRef]

42. Shao, H.D.; Lin, J.; Zhang, L.W.; Galar, D.; Kumar, U. A novel approach of multisensory fusion to collaborative fault diagnosis in maintenance. Inf. Fusion 2021, 74, 65-76. [CrossRef]

43. Wrzochal, M.; Adamczak, S. The problems of mathematical modelling of rolling bearing vibrations. Bull. Pol. Acad. Sci. Tech. Sci. 2020, 68, 1363-1372.

44. Luo, C.; Jia, M.; Wen, Y. The diagnosis approach for rolling bearing fault based on Kurtosis criterion EMD and Hilbert envelope spectrum. In Proceedings of the2017 IEEE 3rd Information Technology and Mechatronics Engineering Conference (ITOEC), Chongqing, China, 3-5 October 2017; pp. 843-847.

45. Available online: https://csegroups.case.edu/bearingdatacenter/pages/welcome-case-western-reserve-university-bearingdata-center-website (accessed on 10 May 2021). 